\title{
Motor Parameterization
}

\author{
Lars Tingelstad*®a and Olav Egeland
}

\begin{abstract}
In this paper, we consider several parameterizations of rigid transformations using motors in 3-D conformal geometric algebra. In particular, we present parameterizations based on the exponential, outer exponential, and Cayley maps of bivectors, as well as a map based on a first-order approximation of the exponential followed by orthogonal projection onto the group manifold. We relate these parameterizations to the matrix representations of rigid transformations in the $3-\mathrm{D}$ special Euclidean group. Moreover, we present how these maps can be used to form retraction maps for use in manifold optimization; retractions being approximations of the exponential map that preserve the convergence properties of the optimization method while being less computationally expensive, and, for the presented maps, also easier to implement.
\end{abstract}

Mathematics Subject Classification. Primary 99Z99; Secondary 00A00.

Keywords. Motion parameterization, Exponential map, Outer exponential map, Cayley map, Retractions.

\section{Introduction}

Traditionally, Lie group methods for optimization, filtering, and integration of rotations and rigid body motions perform the state update using the exponential map, see $[14,20,23,26]$. However, there exist alternative parameterizations and maps that approximate the exponential map which are less computationally expensive. In terms of Riemannian optimization, such maps are called retraction maps $[2,7,29]$; the choice of which is important for highperformance numerical algorithms on nonlinear manifolds [2].

Retraction-based line-search and trust-region optimization methods on Riemannian matrix manifolds are presented in detail in $[1,2,4,31]$; these approaches is based on locally linearizing the manifold around the current iterate, computing the update direction in the current tangent space, and then mapping the solution back to the manifold using the retraction map.

This work was partially funded by the Norwegian Research Council, 237896 SFI Offshore Mechatronics.

${ }^{*}$ Corresponding author. 
Note that, as the tangent space of a nonlinear manifold is a linear space, the use of retractions enable vector space optimization methods on manifolds.

An example of a retraction map is the Cayley map; a rational polynomial map from the Lie algebra to the Lie group. The use of rational polynomial maps is advantageous in numerical applications as it does not involve the evaluation of possibly time-consuming trigonometric and exponential functions [17,24]. In [8], the Cayley map was used in numerical integration of rotations on the special orthogonal group $S O(3)$, see also [16]. Following [3], other retractions on $S O(3)$ can be formed using QR-, polar-, and singular value decomposition.

In $[27,28]$, we employed the exponential map in optimization of rigid body motions, parameterized using motors in 3-D conformal geometric algebra, from observations of geometric objects such as points, lines, circles, and planes. In [6], the authors used observations from lines to estimate motors using the so called motor extended Kalman filter.

In this paper, we present the exponential map and three alternative maps which can be used to form retraction maps for use in manifold optimization of motors. Two of the presented maps are based on the two classical maps: the Cayley map and the outer exponential map. The third map is a novel map constructed by performing a first-order series approximation of the exponential map followed by an orthogonal projection onto the motor manifold. For each of the presented maps, we present how the rotation and translation parameters relate to the rotation and translation distance of the exponential map. In addition, we present how the exponential map and outer exponential map for motors are related to the exponential map and Cayley map for homogeneous transformation matrices in $S E(3)$, respectively.

This paper is organized as follows. Section 2 presents motors in conformal geometric algebra. Section 3 presents screw motions and the exponential map. Presentations of the Cayley map and the outer exponential map follows in Sects. 4 and 5, respectively. Then, Sect. 6, presents a novel projection-based map. Section 7 presents retractions and retractions-based optimization on the motor manifold. Section 8 concludes the paper.

\section{Motors in 3-D Conformal Geometric Algebra}

Let $\mathbb{R}_{4,1}$ denote the conformal geometric algebra of Euclidean 3 -D space $\mathbb{R}^{3}$ generated by the basis vectors $\mathbf{e}_{1}, \mathbf{e}_{2}, \mathbf{e}_{3}, n_{o}, n_{\infty}$ where

$$
\mathbf{e}_{i}^{2}=1, \quad n_{o}^{2}=0, \quad n_{\infty}^{2}=0, \quad \text { and } n_{o} \cdot n_{\infty}=-1
$$

The three Euclidean basis vectors $\mathbf{e}_{i}$ form an orthonormal frame, while the geometric interpretation of the two basis vectors $n_{o}$ and $n_{\infty}$ is that $n_{o}$ represents an arbitrary origin, and $n_{\infty}$ represents the point at infinity.

Following $[6,30]$, let the 8 -D space $\mathbb{M} \subset \mathbb{R}_{4,1}^{+}$be defined by

$$
\mathbb{M}=\operatorname{span}\left\{1, \mathbf{e}_{23}, \mathbf{e}_{31}, \mathbf{e}_{12}, \mathbf{e}_{1} n_{\infty}, \mathbf{e}_{2} n_{\infty}, \mathbf{e}_{3} n_{\infty}, \mathbf{I}_{3} n_{\infty}\right\},
$$

where $\mathbf{I}_{3} \in \mathbb{R}_{3}^{3}$ is the unit Euclidean pseudoscalar. The space $\mathbb{M}$ is closed under the geometric product; if $X, Y \in \mathbb{M}$, then $X Y \in \mathbb{M}$. If $X \in \mathbb{M}$, then 
$\langle X \tilde{X}\rangle_{2}=0$ and $\langle X \tilde{X}\rangle_{4}^{2}=0$, where $\langle\cdot\rangle_{k}$ denotes the grade projection operator onto the grade $k$. The inverse of an element $X \in \mathbb{M}$ is given by

$$
X^{-1}=\tilde{X}\left(\frac{\langle X \tilde{X}\rangle-\langle X \tilde{X}\rangle_{4}}{\langle X \tilde{X}\rangle^{2}}\right)
$$

Note that $X$ is invertible if and only if $|X| \neq 0$, where the absolute $|X|$ of $X$ can be found as the square root of $|X|^{2}=|\langle X \tilde{X}\rangle|=\langle X \tilde{X}\rangle$.

Further, let the 6 -D bivector subspace $\mathbb{B} \subset \mathbb{M}$ be defined by

$$
\mathbb{B}=\operatorname{span}\left\{\mathbf{e}_{23}, \mathbf{e}_{31}, \mathbf{e}_{12}, \mathbf{e}_{1} n_{\infty}, \mathbf{e}_{2} n_{\infty}, \mathbf{e}_{3} n_{\infty}\right\}
$$

and the 2-D subspace $\mathbb{S} \subset \mathbb{M}$ of grades 0 and 4 be defined by

$$
\mathbb{S}=\operatorname{span}\left\{1, \mathbf{I}_{3} n_{\infty}\right\}
$$

An element $X \in \mathbb{M}$ can be split into symmetric and anti-symmetric parts according to $X=S+B$, where $S=\frac{1}{2}(X+\widetilde{X}) \in \mathbb{S}$ is symmetric in that $S=\widetilde{S}$ and $B=\frac{1}{2}(X-\widetilde{X}) \in \mathbb{B}$ is anti-symmetric in that $B=-\widetilde{B}$.

Motors $M \in \mathcal{M}$ model rigid-body motions, where $\mathcal{M}$ is the 6 -D Lie group defined by

$$
\mathcal{M}=\{M \in \mathbb{M}: M \widetilde{M}=1\}
$$

Note that the constraint $M \widetilde{M}=1$ means that $\langle M \widetilde{M}\rangle=1$ and $\langle M \widetilde{M}\rangle_{4}=0$.

Consider the trajectory $M(t): \mathbb{R} \rightarrow \mathcal{M}$ describing a continuous rigid body motion. Let $M(0)=M$, then $\dot{M}(0)$ is said to be a tangent vector to $\mathcal{M}$ at $M$. The set of all tangent vectors at $M$, denoted $\mathrm{T}_{M} \mathcal{M}$, is called the tangent space of $\mathcal{M}$ at $M$, and is of the form

$$
\mathrm{T}_{M} \mathcal{M}=\{M B: B \in \mathbb{B}, M \in \mathcal{M}\}
$$

This can be seen by differentiating the group constraint $\widetilde{M}(t) M(t)=1$ with respect to $t$ and evaluating at $t=0$. It follows that $\widetilde{M} \dot{M}=-\dot{\widetilde{M}} M$. It is seen that $\widetilde{M} \dot{M}$ equals minus its reverse, so it can only contain elements of grade $2,6,10$, etc [12]. That is, there exists a bivector $B \in \mathbb{B}$ such that $\dot{M}=M B$ $[5]$.

The tangent space at the identity, the Lie algebra of $\mathcal{M}$, can be identified as the linear bivector space $\mathbb{B}$ together with the commutator product $B_{1} \times$ $B_{2}=\frac{1}{2}\left(B_{1} B_{2}-B_{2} B_{1}\right)$ for $B_{1}, B_{2} \in \mathbb{B}$.

\subsection{The Dual Unit}

We define the grade 4 blade $\epsilon \in \mathbb{S}$ by

$$
\epsilon=\mathbf{I}_{3}^{-1} n_{\infty}
$$

The blade $\epsilon$ satisfies $\epsilon^{2}=0$, and, as $\epsilon \in \mathbb{S}$, it is symmetric; $\epsilon=\widetilde{\epsilon}$. Moreover, $\epsilon$ anti-commutes with Euclidean vectors $\mathbf{a} \in \mathbb{R}_{3}^{1}$ and commutes with Euclidean 2-blades $\mathbf{B} \in \mathbb{R}_{3}^{2}: \epsilon \mathbf{a}=-\mathbf{a} \epsilon$ and $\epsilon \mathbf{B}=\mathbf{B} \epsilon$, respectively.

We call $\epsilon$ the dual unit, as it is isomorphic to the dual unit in screw theory [21], dual quaternions [25], motor algebra [5,6], as well as to Clifford's $\omega$ introduced in the seminal paper "Preliminary Sketch of Biquaternions" $[10]$. 
It will be shown in the following that multiplication by $\epsilon$ can be seen as a duality operation which transforms a rotation about any axis into a translation along that axis. Note that this property is in direct agreement with Clifford's use of $\omega$, see [10, p. 385].

\section{The Exponential Map}

Any motor $M \in \mathcal{M}$ can be written as the exponential series of a bivector $B \in \mathbb{B}$,

$$
\exp : \mathbb{B} \rightarrow \mathcal{M}: B \mapsto 1+B+\frac{1}{2 !} B^{2}+\frac{1}{3 !} B^{3}+\cdots
$$

Chasles' theorem $[9,11]$ states that a general displacement, or rigid body motion, from one location to another can be achieved by a rotation about a unique axis, and, independently, a translation along that axis. Such a motion is said to be a screw motion about a screw axis, with the property that the rotation and translation commutes. Note that rigid body motions do not generally commute. Let $B_{1}, B_{2} \in \mathbb{B}$, then $\exp \left(B_{1}\right) \exp \left(B_{2}\right)=\exp \left(B_{1}+B_{2}\right)=$ $\exp \left(B_{2}+B_{1}\right)=\exp \left(B_{2}\right) \exp \left(B_{1}\right)$ if and only if $B_{1} \times B_{2}=0$.

Consider a screw motion about a screw axis. Let $\theta \in \mathbb{R}$ be the rotation angle around the screw axis, and let $d \in \mathbb{R}$ be the translation distance along the screw axis, then $\hat{\theta}=\theta+\epsilon d \in \mathbb{S}$ is said to be the dual angle of the screw motion. Further, let the screw axis be given by the dual line $\ell=\mathbf{A}+\epsilon \mathbf{B} \in \mathbb{B}$. Here, $\mathbf{A} \in \mathbb{R}_{3}^{2},|\mathbf{A}|=1$, is a unit Euclidean 2-blade representing the direction of the line, while $\mathbf{B} \in \mathbb{R}_{3}^{2}$ is a Euclidean 2-blade orthogonal to $\mathbf{A}$, in that $\mathbf{A} \cdot \mathbf{B}=0$, representing the moment of the line. Then, the bivector

$$
B=\hat{\theta} \ell \in \mathbb{B}
$$

is said to be a screw.

Any bivector $B \in \mathbb{B}$ of the form $B=\mathbf{B}+\mathbf{t} n_{\infty}$ can be decomposed into screw form. Let the Euclidean vector $\mathbf{t} \in \mathbb{R}_{3}^{1}$ be decomposed into components parallel and perpendicular to the unit Euclidean 2-blade $\mathbf{B} /|\mathbf{B}|$, $\mathbf{t}_{\|}=\mathrm{P}_{\mathbf{B} /|\mathbf{B}|}(\mathbf{t})$ and $\mathbf{t}_{\perp}=\mathbf{t}-\mathbf{t}_{\|}$, respectively. The bivector $B$ can then be written as the sum $B=B_{1}+B_{2}$ where $B_{1}=\mathbf{B}+\mathbf{t}_{\|} n_{\infty}$ and $B_{2}=\mathbf{t}_{\perp} n_{\infty}$ commute. We identify the dual angle $\hat{\theta} \in \mathbb{S}$ of the screw motion of $B$ as $\hat{\theta}=|\mathbf{B}|+\epsilon\left|\mathbf{t}_{\perp}\right|$ and the screw axis $\ell \in \mathbb{B}$ as the dual line $\ell=\left(\mathbf{B}+\mathbf{t}_{\|} n_{\infty}\right) /|\mathbf{B}|$.

If the screw $B \in \mathbb{B}$ is given as in (5), then

$$
\exp \frac{B}{2}=\cos \frac{\theta}{2}+\sin \frac{\theta}{2} \mathbf{A}+\epsilon \frac{d}{2} \cos \frac{\theta}{2} \mathbf{A}+\epsilon \sin \frac{\theta}{2} \mathbf{B}-\epsilon \frac{d}{2} \sin \frac{\theta}{2}
$$

Using that

$$
\cos \frac{\hat{\theta}}{2}=\cos \frac{\theta}{2}-\epsilon d \sin \frac{\theta}{2} \text { and } \sin \frac{\hat{\theta}}{2}=\sin \frac{\theta}{2}+\epsilon \frac{d}{2} \cos \frac{\theta}{2}
$$

the bivector exponential in (6) can be further written as

$$
\exp \frac{B}{2}=\cos \frac{\hat{\theta}}{2}+\sin \frac{\hat{\theta}}{2} \ell
$$


This result can be shown by noting that

$$
\ell^{2}=-1 \quad \text { and } \quad \hat{\theta}^{n}=\theta^{n}+n \theta^{n-1} \epsilon d
$$

and performing a series expansion of the exponential, using that

$$
\cos \hat{\theta}=1-\frac{1}{2 !} \theta^{2}+\frac{1}{4 !} \theta^{4}-\cdots \quad \text { and } \quad \sin \hat{\theta}=\hat{\theta}-\frac{1}{3 !} \hat{\theta}^{3}+\frac{1}{5 !} \hat{\theta}^{3}-\cdots
$$

Now, let the screw $B \in \mathbb{B}$ represent a pure rotation around a line that does not intersect the origin. Then $B=\theta \ell$, with rotation angle $\theta \in \mathbb{R}$ and screw axis $\ell \in \mathbb{B}$. The exponential of $B$ then gives

$$
\exp \frac{\theta}{2} \ell=\cos \frac{\theta}{2}+\sin \frac{\theta}{2} \ell
$$

If the screw axis $\ell$ intersects the origin, then the screw exponential reduces to the rotator (rotation rotor),

$$
\exp \frac{\theta}{2} \mathbf{A}=\cos \frac{\theta}{2}+\sin \frac{\theta}{2} \mathbf{A}
$$

If the rotation angle $\theta=0$ and the translation distance is given by $d \in \mathbb{R}$, then the exponential

$$
\exp \epsilon \frac{d}{2} \ell=1+\frac{1}{2} \mathbf{t} n_{\infty}
$$

reduces to a translator (translation rotor), representing a pure translation, where $\mathbf{t}=d \mathbf{A I}_{3}^{-1} \in \mathbb{R}_{3}^{1}$ is a Euclidean translation vector, encoding a translation of distance $d$ in the direction normal to the rotation plane $\mathbf{A}$.

From (10) and (11), it is seen that the dual unit $\epsilon$ transforms a rotation around an axis into a translation along that axis.

Implementation-wise, the exponential map in (4) can be computed by explicitly evaluating the exponential series up to a certain order [13]. For general multivectors, this approach tends to be slower and less precise than evaluating a closed-form solution. Fortunately, as shown, the exponential map for bivectors $B \in \mathbb{B}$ in (4) has a closed-form solution; see e.g. [27] for an implementation in $\mathrm{C}++$.

\subsection{Matrix Representations}

Let the motor $M \in \mathcal{M}$ be given as the exponential of the bivector $B \in \mathbb{B}$ as $M=\exp (-B / 2)$. Moreover, let $\underline{M}$ be the orthogonal transformation of the multivector $X \in \mathbb{R}_{4,1}$ defined by

$$
\underline{M} X=M X \widetilde{M}
$$

Then, the matrix form $[M]$ of $\underline{M}$ can be written as the matrix exponential

$$
[M]=\exp ([A])
$$

where $[A]$ is the matrix form of the anti-symmetric transformation $\underline{A}$ defined by

$$
\underline{A} X=X \times B
$$

see [22, pp. 158-160]. If the bivector $B$ is given in terms of its components as

$$
B=a_{1} \mathbf{e}_{23}+a_{2} \mathbf{e}_{31}+a_{3} \mathbf{e}_{12}+b_{1} \mathbf{e}_{1} n_{\infty}+b_{2} \mathbf{e}_{2} n_{\infty}+b_{3} \mathbf{e}_{3} n_{\infty}
$$


and $X$ is a flat point expressed on the basis $\left\{\mathbf{e}_{1} \wedge n_{\infty}, \mathbf{e}_{2} \wedge n_{\infty}, \mathbf{e}_{3} \wedge n_{\infty}, n_{o} \wedge\right.$ $\left.n_{\infty}\right\}$, the matrix form $[A]$ of $\underline{A}$ is found to be

$$
[A]=\left[\begin{array}{cccc}
0 & -a_{3} & a_{2} & b_{1} \\
a_{3} & 0 & -a_{1} & b_{2} \\
-a_{2} & a_{1} & 0 & b_{3} \\
0 & 0 & 0 & 0
\end{array}\right]
$$

It is seen that $[A] \in \mathfrak{s e}(3)$, where $\mathfrak{s e}(3)$ is the Lie algebra of the 3 -D special Euclidean group $S E(3)$, and it follows that $[M] \in S E(3)$ is a $4 \times 4$ homogeneous transformation matrix. Moreover, if $X$ is expressed on the basis of the bivector space $\mathbb{B}$, it can easily be shown that $[A]$ and $[M]$ are elements of the $6 \times 6$ adjoint representation of $\mathfrak{s e}(3)$ and $S E(3)$, respectively.

\section{The Cayley Map}

A classic rational polynomial map that can be used as an approximation of the exponential map in Lie-group methods is the Cayley map; which has the advantage of being easier to implement and cheaper to compute than the bivector exponential map presented in the previous section. The Cayley map for dual quaternions was studied in [25], while the Cayley map for 3-D conformal transformations was studied in [17].

The Cayley map for bivectors $B \in \mathbb{B}$ to the group of motors $\mathcal{M}$ is defined as

$$
\text { Cay }: \mathbb{B} \rightarrow \mathcal{M}: B \mapsto(1+B)(1-B)^{-1}
$$

While the exponential map in (4) results, physically, in a screw motion, it is not clear from (14) what the physically resulting motion of the Cayley map is. The relation between the exponential and Cayley maps for motors in terms of the rotation angle, translation distance, and screw axis will be presented in the following.

Let the bivector $B \in \mathbb{B}$ be given in screw form with rotation parameter $\varphi \in \mathbb{R}$, translation parameter $\lambda \in \mathbb{R}$, and screw axis $\ell=\mathbf{A}+\epsilon \mathbf{B},|\mathbf{A}|=1$, and $\mathbf{A} \cdot \mathbf{B}=0$, so that $B=\hat{\varphi} \ell=\varphi \mathbf{A}+\varphi \epsilon \mathbf{B}+\lambda \epsilon \mathbf{A}$. Using the inverse in (1), the Cayley map in (14) can be written as

$$
\text { Cay } B=\frac{(1+B)^{2}\left(1-\left\langle B^{2}\right\rangle+\left\langle B^{2}\right\rangle_{4}\right)}{\left(1-\left\langle B^{2}\right\rangle\right)^{2}}
$$

We first expand $(1+B)^{2}$ and write

$$
(1+B)^{2}=1-\varphi^{2}+2(\varphi \mathbf{A}+\varphi \epsilon \mathbf{B}+\lambda \epsilon \mathbf{A})-2 \epsilon \lambda \varphi
$$

where we have used that

$$
B^{2}=\left\langle B^{2}\right\rangle+\left\langle B^{2}\right\rangle_{4}=-\varphi^{2}-2 \epsilon \lambda \varphi
$$

which follows directly from (9). Then, using that

$$
1-\left\langle B^{2}\right\rangle+\left\langle B^{2}\right\rangle_{4}=1+\varphi^{2}-2 \epsilon \lambda \varphi \quad \text { and } \quad\left(1-\left\langle B^{2}\right\rangle\right)^{2}=\left(1+\varphi^{2}\right)^{2}
$$


the expression in (15) can further be written as

$$
\text { Cay } B=\frac{\left(1-\varphi^{2}+2(\varphi \mathbf{A}+\varphi \epsilon \mathbf{B}+\lambda \epsilon \mathbf{A})-2 \epsilon \lambda \varphi\right)\left(1+\varphi^{2}-2 \epsilon \lambda \varphi\right)}{\left(1+\varphi^{2}\right)\left(1+\varphi^{2}\right)}
$$

and expanded to

$$
\begin{aligned}
\text { Cay } B= & \frac{1-\varphi^{2}}{1+\varphi^{2}} \\
& +\frac{2 \varphi \mathbf{A}}{1+\varphi^{2}}+\frac{2 \varphi \epsilon \mathbf{B}}{1+\varphi^{2}}-\frac{2 \varphi \mathbf{A}}{1+\varphi^{2}} \frac{2 \epsilon \lambda \varphi}{1+\varphi^{2}}+\frac{2 \lambda \epsilon \mathbf{A}}{1+\varphi^{2}} \frac{1+\varphi^{2}}{1+\varphi^{2}} \\
& -\frac{2 \epsilon \lambda \varphi}{1+\varphi^{2}}-\frac{1-\varphi^{2}}{1+\varphi^{2}} \frac{2 \epsilon \lambda \varphi}{1+\varphi^{2}}
\end{aligned}
$$

where

$$
-\frac{2 \varphi \mathbf{A}}{1+\varphi^{2}} \frac{2 \epsilon \lambda \varphi}{1+\varphi^{2}}+\frac{2 \lambda \epsilon \mathbf{A}}{1+\varphi^{2}} \frac{1+\varphi^{2}}{1+\varphi^{2}}=\lambda \frac{2}{1+\varphi^{2}} \frac{1-\varphi^{2}}{1+\varphi^{2}} \epsilon \mathbf{A}=\frac{\lambda}{\varphi} \frac{2 \varphi}{1+\varphi^{2}} \frac{1-\varphi^{2}}{1+\varphi^{2}} \epsilon \mathbf{A}
$$

Finally, this means that

$$
\text { Cay } \begin{aligned}
B= & \frac{1-\varphi^{2}}{1+\varphi^{2}} \\
& +\frac{2 \varphi}{1+\varphi^{2}} \mathbf{A}+\frac{2 \varphi}{1+\varphi^{2}} \epsilon \mathbf{B}+\frac{\lambda}{\varphi} \frac{2 \varphi}{1+\varphi^{2}} \frac{1-\varphi^{2}}{1+\varphi^{2}} \epsilon \mathbf{A} \\
& -\lambda \frac{2 \varphi}{1+\varphi^{2}} \epsilon-\lambda \frac{1-\varphi^{2}}{1+\varphi^{2}} \frac{2 \varphi}{1+\varphi^{2}} \epsilon
\end{aligned}
$$

To compare the Cayley map with the exponential map, we substitute the rotation parameter $\varphi$ with

$$
\varphi=\tan \frac{\theta}{4}=\frac{\sin \theta / 2}{1+\cos \theta / 2}
$$

The rotation parameter $\varphi$ then satisfies the trigonometric identities

$$
\frac{1-\varphi^{2}}{1+\varphi^{2}}=\cos \frac{\theta}{2} \text { and } \quad \frac{2 \varphi}{1+\varphi^{2}}=\sin \frac{\theta}{2}
$$

for rotation angle $\theta \in \mathbb{R}$, resulting in

$$
\begin{aligned}
\operatorname{Cay}_{\mathcal{M}} B= & \cos \frac{\theta}{2}+\sin \frac{\theta}{2} \mathbf{A}+\sin \frac{\theta}{2} \epsilon \mathbf{B}+\lambda \cos \frac{\theta}{2}\left(1+\cos \frac{\theta}{2}\right) \epsilon \mathbf{A} \\
& -\lambda \sin \frac{\theta}{2}\left(1+\cos \frac{\theta}{2}\right) \epsilon
\end{aligned}
$$

Finally, we substitute the translation parameter $\lambda$ with

$$
\lambda=\frac{d / 4}{\cos ^{2} \theta / 4}=\frac{d / 2}{1+\cos \theta / 2}
$$

where $d \in \mathbb{R}$ is the translation distance. Using the substitutions in (18) and (19), the bivector $B$ can be written in terms of the dual angle $\hat{\theta}=\theta+\epsilon d$ as

$$
B=\tan \frac{\hat{\theta}}{4} \ell
$$


which follows directly from the expressions in (1) and (7). It can then be seen that the Cayley map for motors satisfies

$$
\text { Cay } \tan \frac{\hat{\theta}}{4} \ell=\exp \frac{\hat{\theta}}{2} \ell
$$

and that for small $\theta$ and small $d$ the approximation

$$
\text { Cay } \frac{\hat{\theta}}{4} \ell \approx \exp \frac{\hat{\theta}}{2} \ell
$$

can be used.

Note that in applications, the Cayley map would be implemented using (14) and the inverse in (1), directly. The above presented expressions are only used to relate the Cayley map geometrically to the exponential map.

\section{The Outer Exponential Map}

Another classical map of interest from the bivector space $\mathbb{B}$ to the motor group $\mathcal{M}$ that can be used as an approximation to the exponential map is the outer (exterior) exponential map; the exponential series of a bivector with the outer product as multiplication [15, 17-19].

Employing the notation in [15], and restricting to bivectors $B \in \mathbb{B}$, the outer exponential map is defined as

$$
\operatorname{Exp}: \mathbb{B} \rightarrow \mathbb{M}: B \mapsto 1+B+\frac{1}{2 !} B \wedge B+\frac{1}{3 !} B \wedge B \wedge B+\cdots
$$

Note that this series is finite and truncates at $\frac{1}{2 !} B \wedge B$ as higher order terms result in elements of grade $6,8,10$, etc; these elements are thus not elements of the space $\mathbb{M}$.

The outer exponential map of a bivector $B \in \mathbb{B}$ results in an even versor $V=\operatorname{Exp} B$ satisfying $V \widetilde{V} \in \mathbb{R}$ and transform multivectors $X \in \mathbb{R}_{4,1}$ according to $X \mapsto V X V^{-1}$. That is, the outer exponential map of a bivector $B \in \mathbb{B}$ must be normalized to form a motor $M \in \mathcal{M}$.

We define the normalized outer exponential by

$$
\operatorname{Exp}_{\mathcal{M}}: \mathbb{B} \rightarrow \mathcal{M}: B \mapsto \frac{\operatorname{Exp} B}{|\operatorname{Exp} B|}
$$

Let the rotation parameter of the bivector $B \in \mathbb{B}$ be given by $\varphi \in \mathbb{R}$, let the translation parameter be given by $\lambda \in \mathbb{R}$, that is, the dual angle of the rigid body motion is given by $\hat{\varphi}=\varphi+\epsilon \lambda$, and, again, let the screw axis be given by the dual line $\ell=\mathbf{A}+\epsilon \mathbf{B}$ where $|\mathbf{A}|=1$ and $\mathbf{A} \cdot \mathbf{B}=0$, giving $B=\hat{\varphi} \ell=\varphi \mathbf{A}+\varphi \epsilon \mathbf{B}+\lambda \epsilon \mathbf{A}$. Using (16) and that $\frac{1}{2 !} B \wedge B=\frac{1}{2}\left\langle B^{2}\right\rangle_{4}$, we find that

$$
\begin{aligned}
\operatorname{Exp} B & =1+B+\frac{1}{2}\left\langle B^{2}\right\rangle_{4} \\
& =1+\varphi \mathbf{A}+\varphi \epsilon \mathbf{B}+\lambda \epsilon \mathbf{A}-\epsilon \lambda \varphi
\end{aligned}
$$

The absolute value $|\operatorname{Exp} B|$ can then be found as the square root of

$$
|\operatorname{Exp} B|^{2}=\operatorname{Exp} B(\operatorname{Exp}-B)=1-\left\langle B^{2}\right\rangle=1+\varphi^{2}
$$


Here, we have used that $\left\langle B^{2}\right\rangle_{4}^{2}=0$ and that $B\left\langle B^{2}\right\rangle_{4}=\left\langle B^{2}\right\rangle_{4} B$, since elements of grade 4 commute with bivectors. The normalized outer exponential $\operatorname{Exp}_{\mathcal{M}} B$ can then be written as

$$
\begin{aligned}
\operatorname{Exp}_{\mathcal{M}} B= & \frac{1}{\sqrt{1+\varphi^{2}}}+\frac{\varphi}{\sqrt{1+\varphi^{2}}} \mathbf{A} \\
& +\frac{\varphi}{\sqrt{1+\varphi^{2}}} \epsilon \mathbf{B}+\lambda \frac{1}{\sqrt{1+\varphi^{2}}} \epsilon \mathbf{A}-\lambda \frac{\varphi}{\sqrt{1+\varphi^{2}}} \epsilon
\end{aligned}
$$

To relate the normalized outer exponential in (23) to the exponential in (6), we substitute the rotation and translation parameters by

$$
\varphi=\tan \frac{\theta}{2} \quad \text { and } \quad \lambda=\frac{d}{2}
$$

respectively. The rotation parameter $\varphi$ then satisfies

$$
\frac{1}{\sqrt{1+\varphi^{2}}}=\cos \frac{\theta}{2} \text { and } \frac{\varphi}{\sqrt{1+\varphi^{2}}}=\sin \frac{\theta}{2}
$$

From (24), it can be seen that the outer exponential map in (23) satisfies

$$
\operatorname{Exp}_{\mathcal{M}}\left(\tan \frac{\theta}{2}+\epsilon \frac{d}{2}\right) \ell=\exp \left(\frac{\theta}{2}+\epsilon \frac{d}{2}\right) \ell
$$

for $\theta \neq \pi \bmod 2 \pi$. Moreover, for small $\theta$ and small $d$ the approximation

$$
\operatorname{Exp}_{\mathcal{M}} \frac{\hat{\theta}}{2} \ell \approx \exp \frac{\hat{\theta}}{2} \ell
$$

can be used, where the dual angle $\hat{\theta} \in \mathbb{S}$ is given by $\hat{\theta}=\theta+\epsilon d$.

The normalized outer exponential map is easy to implement. Given the bivector $B=\mathbf{A}+\epsilon \mathbf{B} \in \mathbb{B}, B^{2}=A^{2}+\epsilon(\mathbf{A B}+\mathbf{B A})=A^{2}+2 \epsilon(\mathbf{A} \cdot \mathbf{B})$ so that $B \wedge B=\left\langle B^{2}\right\rangle_{4}=2 \epsilon(\mathbf{A} \cdot \mathbf{B})$. This means that

$$
\operatorname{Exp}_{\mathcal{M}} B=\frac{1+\mathbf{A}+\epsilon \mathbf{B}+\epsilon \mathbf{A} \cdot \mathbf{B}}{|1+\mathbf{A}|}
$$

\subsection{Matrix Representation}

Let the motor $U \in \mathcal{M}$ be given as the outer exponential of the bivector $B \in \mathbb{B}$ as $U=\operatorname{Exp}_{\mathcal{M}} B$ and let $\underline{U}$ be the orthogonal transformation of the multivector $X \in \mathbb{R}_{4,1}$ defined by

$$
\underline{U} X=U X \widetilde{U}
$$

Then, the matrix form $[U]$ of $\underline{U}$ can be written as the Cayley map

$$
[U]=(1+[A])(1-[A])^{-1}
$$

where $[A]$ is the matrix form of the anti-symmetric transformation $\underline{A}$ defined in (12). See also [18]. If, again, $X$ is represented on the basis $\left\{\mathbf{e}_{1} \wedge n_{\infty}, \mathbf{e}_{2} \wedge\right.$ $\left.n_{\infty}, \mathbf{e}_{3} \wedge n_{\infty}, n_{o} \wedge n_{\infty}\right\}$, then $[A] \in \mathfrak{s e}(3)$ and $[U] \in S E(3)$. The Cayley map for the $4 \times 4$ and $6 \times 6$ representation of $S E(3)$ are presented in detail in [24]. 


\section{Orthogonal Projection}

The exponential, Cayley, and exterior exponential maps are all known classical maps. A novel map from the bivector space $\mathbb{B}$ to the motor group $\mathcal{M}$ can be formed by performing a first-order series expansion of the exponential map followed by orthogonal projection onto the motor manifold.

In the following we consider in detail the map defined by

$$
\operatorname{Proj}_{\mathcal{M}}: \mathbb{B} \rightarrow \mathcal{M}: B \mapsto \mathrm{P}_{\mathcal{M}}(1+B)
$$

where

$$
\mathrm{P}_{\mathcal{M}}: \mathbb{M} \rightarrow \mathcal{M}: X \mapsto \frac{X}{|X|}\left(1-\frac{\langle X \tilde{X}\rangle_{4}}{2\langle X \tilde{X}\rangle}\right)
$$

is the orthogonal projection based on polar decomposition presented in [30, Lemma 2.3].

Again, let the bivector $B \in \mathbb{B}$ be given by $B=(\varphi+\epsilon \lambda) \ell$ where $\varphi \in \mathbb{R}$ and $\lambda \in \mathbb{R}$ are the rotation and translation parameters, respectively, and $\ell=\mathbf{A}+\epsilon \mathbf{B} \in \mathbb{B}$ is the screw axis, where $|\mathbf{A}|=1$ and $\mathbf{A} \cdot \mathbf{B}=0$. This gives $B=\hat{\varphi} \ell=\varphi \mathbf{A}+\varphi \epsilon \mathbf{B}+\lambda \epsilon \mathbf{A}$. Let $X=1+B$, and using (16), we find that

$$
X \tilde{X}=1-\left\langle B^{2}\right\rangle-\left\langle B^{2}\right\rangle_{4}=1+\varphi^{2}+2 \lambda \varphi \epsilon
$$

The absolute value $|X|$ of $X$ follows directly,

$$
|X|=\sqrt{1+\varphi^{2}}
$$

The map $\operatorname{Proj}_{\mathcal{M}} B$ can then be written as

$$
\operatorname{Proj}_{\mathcal{M}} B=\frac{(1+\varphi \mathbf{A}+\epsilon \varphi \mathbf{B}+\epsilon \lambda \mathbf{A})}{\sqrt{1+\varphi^{2}}}\left(1-\frac{\lambda \varphi \epsilon}{1+\varphi^{2}}\right)
$$

which can be expanded to

$$
\begin{aligned}
\operatorname{Proj}_{\mathcal{M}} B= & \frac{1}{\sqrt{1+\varphi^{2}}}+\frac{\varphi}{\sqrt{1+\varphi^{2}}} \mathbf{A}+\frac{\varphi}{\sqrt{1+\varphi^{2}}} \epsilon \mathbf{B} \\
& +\lambda\left(\frac{1}{\sqrt{1+\varphi^{2}}}-\frac{\varphi^{2}}{\sqrt[3]{1+\varphi^{2}}}\right) \epsilon \mathbf{A}-\frac{\lambda \varphi \epsilon}{\sqrt[3]{1+\varphi^{2}}}
\end{aligned}
$$

By substituting the rotation and translation parameters with

$$
\varphi=\tan \frac{\theta}{2} \text { and } \lambda=\frac{d}{2}
$$

respectively, and using (24), the final expression for the projection-based map $\operatorname{Proj}_{\mathcal{M}}$ becomes

$$
\begin{aligned}
\operatorname{Proj}_{\mathcal{M}} B= & \cos \frac{\theta}{2}+\sin \frac{\theta}{2} \mathbf{A}+\sin \frac{\theta}{2} \epsilon \mathbf{B}+\frac{d}{2} \cos \frac{\theta}{2}\left(1-\sin ^{2} \frac{\theta}{2}\right) \epsilon \mathbf{A} \\
& -\frac{d}{2} \sin \frac{\theta}{2}\left(1-\sin ^{2} \frac{\theta}{2}\right) \epsilon
\end{aligned}
$$

It is seen that for small $\theta$ and small $d$ the approximation

$$
\mathrm{P}_{\mathcal{M}} \frac{\hat{\theta}}{2} \ell \approx \exp \frac{\hat{\theta}}{2} \ell
$$


can be used for dual angle $\hat{\theta}=\theta+\epsilon d \in \mathbb{S}$.

Note that it is also possible to perform higher-order series expansion of the exponential map followed by orthogonal projection onto the motor manifold; however, this has not been investigated further at the present stage.

\section{Retraction-Based Optimization on the Motor Manifold}

Consider the following optimization problem

$$
\min _{M \in \mathcal{M}} f(M)
$$

where $f: \mathcal{M} \rightarrow \mathbb{R}$ is a smooth real valued function to be minimized and $M \in \mathcal{M}$ is a motor. A retraction map is a map from the tangent space $\mathrm{T}_{M} \mathcal{M}$ to the motor manifold $\mathcal{M}$ defined by

$$
\operatorname{Retr}_{M}: \mathrm{T}_{M} \mathcal{M} \rightarrow \mathcal{M}: X \mapsto \operatorname{Retr}_{M} X
$$

where $\operatorname{Retr}_{M}$ is continuously differentiable, satisfies $\operatorname{Retr}_{M}(0)=M$, and for every tangent vector $X \in \mathrm{T}_{M} \mathcal{M}$ there exists a curve $\gamma_{X}: t \mapsto \operatorname{Retr}_{M}(t X)$ such that $\dot{\gamma}_{X}(0)=X$, see [31] and [2, Def. 2.31 and Def. 4.11], respectively. The retraction $\operatorname{Retr}_{M}$ maps a tangent vector $X \in \mathrm{T}_{M} \mathcal{M}$ back to the manifold, as well as moves the cost function $f$ from the manifold $\mathcal{M}$ to the tangent space $\mathrm{T}_{M} \mathcal{M}$ by evaluating the composite function

$$
\widehat{f}_{M}: \mathrm{T}_{M} \mathcal{M} \rightarrow \mathbb{R}: X \mapsto f\left(\operatorname{Retr}_{M}(X)\right)
$$

The tangent space $\mathrm{T}_{M} \mathcal{M}$ is a six-dimensional linear space. This means that, by moving the cost function $f$ to the linear space $\mathrm{T}_{M} \mathcal{M}$, the retraction $\operatorname{Retr}_{M}$ enables the use of vector space optimization methods on the manifold.

Let $M_{k} \in \mathcal{M}$ be the iterate in iteration $k$ of a numerical optimization method solving (28), e.g., gradient descent or other line-search or trust-region methods. The search direction for the next iterate $M_{k+1} \in \mathcal{M}$ can then be computed in the tangent space $\mathrm{T}_{M_{k}} \mathcal{M}$ as the tangent vector $X \in \mathrm{T}_{M_{k}} \mathcal{M}$, and mapped back to the manifold by the update equation

$$
M_{k+1}=\operatorname{Retr}_{M_{k}}(\alpha X)
$$

where $\alpha \in \mathbb{R}$ is the step length.

The update Eq. (29) can be considered as moving along a curve $\operatorname{Retr}_{X}$ in the direction of the tangent vector $X$ while constrained to the motor manifold $\mathcal{M}[2]$.

The exponential map can be used to form a retraction on the motor manifold; the exponential retraction. However, one of the main ideas behind retraction-based optimization is to approximate the exponential with other maps that are less computationally expensive while preserving the convergence properties of the optimization method $[2,4]$. The above presented maps from the bivector space $\mathbb{B}$ (the tangent space at the identity) to the motor group $\mathcal{M}$ can all be used to form such retraction maps for use in optimization. 


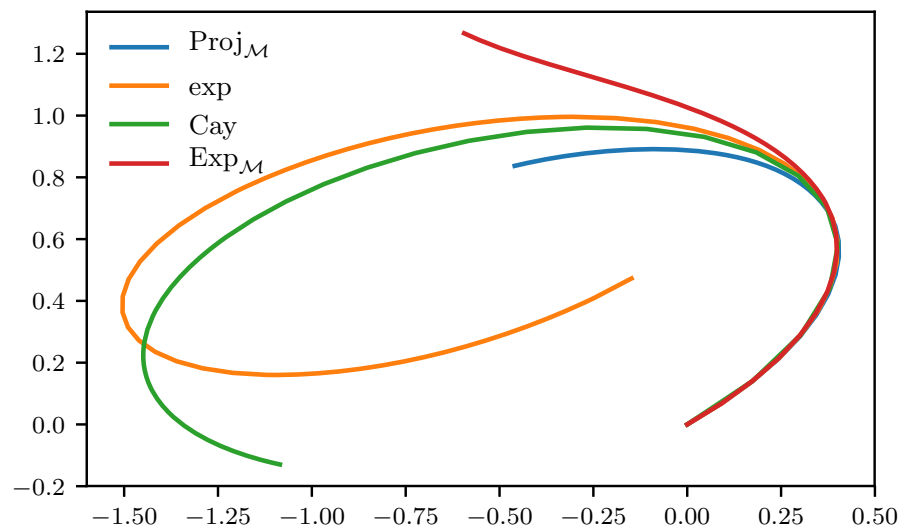

Figure 1. Projection onto the $y z$-plane of the trajectories formed by the presented maps, given a random unit bivector $B \in \mathbb{B}$, when the rotation angle $\theta \in \mathbb{R}$ is in the range $0 \leq$ $\theta<\pi$

Let $M \in \mathcal{M}$ and $X=M B \in \mathrm{T}_{M} \mathcal{M}$. We define the exponential retraction by

$$
\exp _{M}: \mathrm{T}_{M} \mathcal{M} \rightarrow \mathcal{M}: X \mapsto M \exp (\widetilde{M} X)=M \exp B
$$

and similarly, the Cayley retraction and the outer exponential retraction by

$$
\operatorname{Cay}_{M}: \mathrm{T}_{M} \mathcal{M} \rightarrow \mathcal{M}: X \mapsto M \operatorname{Cay}(\widetilde{M} X)=M \text { Cay } B
$$

and

$$
\operatorname{Exp}_{M} X: \mathrm{T}_{M} \mathcal{M} \rightarrow \mathcal{M}: X \mapsto M \operatorname{Exp}_{\mathcal{M}}(\widetilde{M} X)=M \operatorname{Exp}_{\mathcal{M}} B
$$

respectively. Further, using that the tangent space $\mathrm{T}_{M} \mathcal{M}$ can be considered as a linear subspace of the motor space $\mathbb{M}$, we define the projection retraction by

$$
\begin{aligned}
& \operatorname{Proj}_{M}: \mathrm{T}_{M} \mathcal{M} \rightarrow \mathcal{M}: X \mapsto \mathrm{P}_{\mathcal{M}}(M+X)=\mathrm{P}_{\mathcal{M}}(M+M B) \\
& \quad=\mathrm{P}_{\mathcal{M}}(M(1+B))=M \mathrm{P}_{\mathcal{M}}(1+B)=M \operatorname{Proj}_{\mathcal{M}} B
\end{aligned}
$$

To compare the presented retraction maps, we consider the motion generated by the update equation in (29), given an update bivector $B \in \mathbb{B}$ with absolute value $|B|=1$ when the step length $\alpha \in \mathbb{R}$ is in the range $0 \leq \alpha<\pi$; in this setting the step length $\alpha$ corresponds to the rotation angle of the generated motor. The trajectories formed by the presented maps are shown in Fig. 1. It is seen that all three alternative maps, as expected, approximate the exponential map for small $\alpha$; small $\alpha$ typically being the case in line-search methods. The main result here is then that, in manifold optimization and in other Lie-group methods, the exponential map can be exchanged with any of the other presented maps which are less computationally expensive and easier to implement. 


\section{Conclusion}

In this paper, we have presented the exponential map, as well as three alternative maps that can be used in Lie group methods such as interpolation, optimization and numerical integration of rigid transformations parameterized by motors in 3-D conformal geometric algebra; namely the Cayley map, the outer exponential map, and a novel map based on a first-order series expansion of the exponential map followed by orthogonal projection onto the motor manifold. We have also shown how the exponential and outer exponential map relate to the exponential and Cayley maps on $S E(3)$, respectively.

Moreover, we have shown how these maps can be used to form retraction maps on the motor manifold that can be used in manifold optimization; retractions being approximations to the exponential map that are easier to compute that do not sacrifice the convergence properties of the optimization method of choice. In this regard, the main result of the paper is that we have shown how these maps relate to and approximate the exponential map for small angles, and that in manifold optimization, the exponential map can be exchanged with any of the presented maps which are computationally less expensive and easier to implement.

Open Access. This article is distributed under the terms of the Creative Commons Attribution 4.0 International License (http://creativecommons.org/licenses/ by/4.0/), which permits unrestricted use, distribution, and reproduction in any medium, provided you give appropriate credit to the original author(s) and the source, provide a link to the Creative Commons license, and indicate if changes were made.

\section{References}

[1] Absil, P.-A., Baker, C.G., Gallivan, K.A.: Trust-region methods on Riemannian manifolds. Found. Comput. Math. 7(3), 303-330 (2007)

[2] Absil, P.-A., Mahony, R., Sepulchre, R.: Optimization Algorithms on Matrix Manifolds. Princeton University Press, Princeton (2008)

[3] Adler, R.L., Dedieu, J.P., Margulies, J.Y., Martens, M., Shub, M.: Newton's method on riemannian manifolds and a geometric model for the human spine. IMA J. Numer. Anal. 22(3), 359-390 (2002)

[4] Baker, C.G.: Riemannian Manifold Trust-Region Methods with Applications to Eigenproblems. PhD thesis, College of Arts and Sciences, Florida State University (2008)

[5] Bayro-Corrochano, E.: Motor algebra approach for visually guided robotics. Pattern Recognit. 35(1), 279-294 (2002)

[6] Bayro-Corrochano, E., Zhang, Y.: The motor extended kalman filter: a geometric approach for rigid motion estimation. J. Math. Imaging Vis. 13(3), 205-228 (2000)

[7] Boumal, N., Mishra, B., Absil, P.-A., Sepulchre, R.: Manopt, a matlab toolbox for optimization on manifolds. J. Mach. Learn. Res. 15, 1455-1459 (2014) 
[8] Celledoni, E., Owren, B.: Lie group methods for rigid body dynamics and time integration on manifolds. Comput. Methods Appl. Mech. Eng. 192(3), 421-438 (2003)

[9] Chasles, M.: Notes sur les Propriétés Générales du Système de Deux Corps Semblades entr'eux. Bulletin de Sciences Mathematiques, Astronomiques Physiques et Chimiques, pp. 321-326 (1830)

[10] Clifford, W.K.: Preliminary sketch of biquaternions. Proc. Lond. Math. Soc. 4, 361-395 (1873)

[11] Davidson, J.K., Hunt, K.H.: Robots and Screw Theory: Applications of Kinematics and Statics to Robotics. Oxford University Press Inc., New York (2004)

[12] Doran, C., Lasenby, A.: Geometric Algebra for Physicists. Cambridge University Press, Cambridge (2003)

[13] Dorst, L., Fontijne, D., Mann, S.: Geometric Algebra for Computer Science: An Object-Oriented Approach to Geometry. Morgan Kaufmann Publishers Inc., San Francisco (2007)

[14] Hairer, E., Lubich, C., Wanner, G.: Geometric Numerical Integration: Structure-Preserving Algorithms for Ordinary Differential Equations. Springer series in computational mathematics. Springer, Berlin (2006)

[15] Helmstetter, J.: Exponentials of bivectors and their symplectic counterparts. Adv. Appl. Clifford Algebras 18(3), 689-698 (2008)

[16] Iserles, A., Munthe-Kaas, H.Z., Nørsett, S.P., Zanna, A.: Lie-group methods. Acta Numer. 9, 215-365 (2000)

[17] Li, H.: Parameterization of 3D Conformal Transformations in Conformal Geometric Algebra. Springer, London, pp. 71-90 (2010)

[18] Lounesto, P.: Cayley transform, outer exponential and spinor norm. In: Proceedings of the Winter School "Geometry and Physics". Circolo Matematico di Palermo, pp. 191-198 (1987)

[19] Lounesto, P.: Clifford Algebras and Spinors. London Mathematical Society lecture note series, 2nd edn. Cambridge University Press, Cambridge (2001)

[20] Ma, Y., Košecká, J., Sastry, S.: Optimization criteria and geometric algorithms for motion and structure estimation. Int. J. Comput. Vis. 44(3), 219-249 (2001)

[21] McCarthy, J.M., Soh, G.S.: Geometric design of linkages. Springer, New York, NY (2011)

[22] Riesz, M.: Clifford Numbers and Spinors (Chapters I-IV). Springer, Dordrecht, pp. 1-196 (1993)

[23] Sarkis, M., Diepold, K.: Camera-Pose estimation via projective Newton optimization on the manifold. IEEE Trans. Image Process. 21(4), 1729-1741 (2012)

[24] Selig, J.M.: Cayley maps for se(3). In: 12th International Federation for the Promotion of Mechanism and Machine Science World Congress, p. 6 (2007)

[25] Selig, J.M.: Exponential and Cayley maps for dual quaternions. Adv. Appl. Clifford Algebras 20(3), 923-936 (2010)

[26] Smith, S.T.: Geometric Optimization Methods for Adaptive Filtering. PhD thesis, Harvard University (1993)

[27] Tingelstad, L., Egeland, O.: Automatic multivector differentiation and optimization. Adv. Appl. Clifford Algebras 27(1), 707-731 (2017) 
[28] Tingelstad, L., Egeland, O.: Motor estimation using heterogeneous sets of objects in conformal geometric algebra. Adv. Appl. Clifford Algebras 27(3), 2035-2049 (2017)

[29] Townsend, J., Koep, N., Weichwald, S.: Pymanopt: a python toolbox for optimization on manifolds using automatic differentiation. J. Mach. Learn. Res. 17(137), 1-5 (2016)

[30] Valkenburg, R., Dorst, L.: Estimating Motors from a Variety of Geometric Data in 3D Conformal Geometric Algebra. In: Dorst, L., Lasenby, J. (eds.) Guide to Geometric Algebra in Practice, pp. 25-45. Springer, London (2011)

[31] Vandereycken, B.: Riemannian and multilevel optimization for rankconstrained matrix problems. PhD thesis, Department of Computer Science, KU Leuven (2010)

Lars Tingelstad and Olav Egeland

Department of Mechanical and Industrial Engineering,

Faculty of Engineering

NTNU, Norwegian University of Science and Technology

7491 Trondheim

Norway

e-mail: lars.tingelstad@ntnu.no

Olav Egeland

e-mail: olav.egeland@ntnu.no

Received: December 31, 2017.

Accepted: March 3, 2018. 